\title{
Vocabulario, narración y argumentación en los primeros años de la infancia y la niñez. Una revisión de investigaciones \\ Vocabulary, narration and argumentation during the first years of infancy and childhood. A research review
}

\author{
Celia Renata Rosemberg, Alejandra Menti, Alejandra Stein, \\ Florencia Alam y Maia Migdalek \\ Consejo Nacional de Investigaciones Científicas y Técnicas, Argentina
}

\begin{abstract}
Resumen
Se presenta una revisión de investigaciones que atienden al desempeño psicolingüístico infantil referido al vocabulario, al discurso narrativo y al argumentativo. En el marco de una amplia presentación de trabajos de investigación en el tema, el foco de la revisión son los trabajos previos realizados en el marco de la línea de investigación que llevamos a cabo en Argentina con niños de diversos grupos socioculturales. Se incluyen, a modo de ilustración de los conceptos, intercambios audio o videofilmados a niños de dos años y seis meses a cinco años en diferentes contextos de interacción en el hogar y en la escuela. En la presentación conceptual, así como en el análisis de los intercambios, se señalan las interrelaciones entre el vocabulario y el discurso narrativo y argumentativo en las interacciones sociales durante la consecusión de las actividades que configuran la vida cotidiana en el hogar, la comunidad y el entorno escolar. Asimismo, se señala la necesidad de atender, desde una perspectiva multimodal, al contexto de interacción social en el que se insertan tempranamente las palabras infantiles cuando los niños comienzan a producir discursos que les permiten realizar su intención comunicativa.
\end{abstract}

Palabras clave: discurso argumentativo, discurso narrativo, infancia, niñez, vocabulario

\begin{abstract}
We present a research review of studies about children's psycholinguistic performance regarding vocabulary as well as narrative and argumentative discourse. Within the framework of a wide array of research studies on the subject, this revision focuses on the line of research that we have been carrying out with children from diverse sociocultural groups in Argentina. In order to illustrate the concepts, we have included audio or video conversational exchanges among children between two and half years and five years old. The exchanges were recorded in different contexts of interaction at school and at home. In the theoretical presentation, as well as in the analysis of exchanges we point out the relationships between vocabulary and narrative and argumentative discourse, that took place within the social interactions in the daily activities at home, in the community, and school environments. Likewise, we highlight the need to attend to the context of social interaction in which a child's vocabulary is inserted early on, when children begin to produce discourse in order to carry out their communicational goals.
\end{abstract}

Keywords: Argumentative discourse, childhood, infancy, narrative discourse, vocabulary

Celia Renata Rosemberg, Alejandra Menti, Alejandra Stein, Florencia Alam, Maia Migdalek; Consejo Nacional de Investigaciones Científicas y Técnicas (CONICET), Argentina.

La correspondencia en relación con este artículo se dirige a Celia Renata Rosemberg, Centro Interdisciplinario de Investigaciones en Psicología Matemática y Experimental (CIIPME-CONICET), Tte. Juan Perón 2158, Ciudad Autónoma de Buenos Aires, Argentina, CP. 1040. Dirección electrónica: crrosem@,hotmail.com 
El presente trabajo aborda el desarrollo del vocabulario, del discurso narrativo y del argumentativo en niños pequeños. La relevancia de atender conjuntamente al vocabulario y al discurso puede ser ponderada en el marco de modelos psicolingüísticos actuales (Nelson, 2007; Tomasello, 2003) que, partiendo de las premisas del constructivismo social (ver Castellaro, 2012), han mostrado que el desarrollo del vocabulario tiene lugar en contextos de interacción en los que el lenguaje es usado tempranamente en la producción discursiva para desarrollar actividades compartidas. Las investigaciones realizadas en la perspectiva de estos modelos asumen que el lenguaje juega un papel preponderante en la organización de los sistemas cognitivo y socioafectivo, y que el contexto posee un papel fundamental en su desarrollo. En este marco, la noción de "contexto" no alude solo al entorno situacional de la actividad, sino que se halla configurado por las interacciones sociales, su textura discursiva, lingüística y multimodal. El contexto es también "contexto cognitivo", en tanto comprende las representaciones, los significados que las personas ponen en juego en los intercambios. El lenguaje posee no solo funciones en la comunicación humana, sino también permite representar y aproximarse heurísticamente al mundo en la textura de las actividades y de la interacción social, el lenguaje contribuye al proceso de desarrollo lingüístico y cognitivo infantil (Nelson, 1996, 2007; Tomasello, 2003). Por ello, cobran importancia las interacciones sociales de las que participan los niños pequeños en el hogar y en la escuela y que dan lugar a la adquisición de vocabulario y al desarrollo de formas y estrategias discursivas (Hess \& Álvarez, 2010; Hess \& Prado, 2013).

Tradicionalmente, desde las primeras investigaciones que, a fines de la década de los años 1960 y comienzos de 1970, atendieron al papel de la interacción en el desarrollo del vocabulario y una década después, a su papel en el desarrollo del discurso infantil, el foco se ha centrado en las interacciones entre el adulto, principalmente la madre y el niño. Estos estudios han sido mayoritariamente realizados con poblaciones urbanas de sectores medios (Farrant \& Reese, 2000; Hudson, 2006; Melzi \& Caspe, 2005; Nelson, 1996; Schöder, Keller \& Kleis, 2013; Snow, 1983; Sparks, Carmiol \& Ríos, 2013) y han tomado como referente el modelo prevalente en estos sectores sociales, en los que la díada madre-niño constituye el núcleo del desarrollo, aun en los casos en los que abordan el estudio en otros grupos sociales (Nolivos \& Leyva, 2013; Sparks, 2008; Wishard Guerra, 2008). Sin embargo, otras investigaciones más recientes llevadas a cabo o bien en poblaciones de sectores medios (Cekaite, Blum-Kulka, Grøver \& Teubal, 2014), o bien en grupos indígenas y poblaciones en situación de pobreza (Rogoff, 2003; Rosemberg, Stein \& Alam, 2013; Stein \& Rosemberg, 2012) han mostrado la frecuencia y la relevancia de las interacciones entre niños, así como su valor específico para el desarrollo discursivo (Alam \& Rosemberg, 2013; Migdalek, Rosemberg \& Santibáñez, 2014; Nicolopoulou, 2008). Estas investigaciones más recientes han comenzado a desplazarse desde el tradicional foco unitario en el modo oral hacia una perspectiva multimodal y contemplar, también, el valor que poseen los gestos, la dirección de la mirada, la entonación, la posición corporal y los objetos presentes, que en los intercambios contribuyen conjuntamente con la información verbal a configurar las situaciones de desarrollo del vocabulario (Liszkowski, Carpenter, Striano \& Tomasello, 2006; Matthews, Behne, Lieven \& Tomasello, 2012; Menti \& Alam, 2014; Menti \& Rosemberg, 2013; Tomasello, Carpenter \& Liszkowski, 2007), y la construcción en colaboración de discurso (Alam \& Rosemberg, 2015; Goodwin, 1997).

Actualmente, el estudio de la adquisición de vocabulario y del desarrollo discursivo ocupa un lugar importante en las investigaciones, por la relevancia que estos aprendizajes revisten en las trayectorias educacionales de los niños; en particular, en relación con la comprensión y la producción de textos orales y escritos (Strasser, Del Río \& Larraín, 2014; Perfetti, 2007) y el acceso al sistema de escritura (Carmiol, Sparks \& Ríos 2013; Goswami, 2003). Con respecto al desarrollo discursivo, en particular de la narración, se ha mostrado su papel destacado no solo en la comunicación 
VOCABULARIO, NARRACIÓN Y ARGUMENTACIÓN EN LOS PRIMEROS AÑOS DE LA INFANCIA Y LA NIÑEZ.

humana y en la estructuración de la cognición (Nelson, 1996, 2007), sino también en la configuración de la identidad individual y comunitaria (Auza-Benavides, 2013; McCabe, Bailey \& Melzi, 2008; Poveda, Morgade \& Alonso, 2009; Wortham, 2001). Otras formas discursivas, como la argumentación, cuya génesis en los primeros años ha sido menos estudiada (Peronard, 1991; Zadunaisky-Ehrlich \& Blum-Kulka, 2010), constituye uno de los discursos preeminentes en la apropiación y la producción de nuevos conocimientos en los trayectos educativos más avanzados.

Resulta, por lo tanto, pertinente la necesidad de un enfoque comprensivo del desarrollo del vocabulario y de distintas formas de discurso en el marco de las interacciones sociales de las que participan tempranamente los niños de distintos grupos sociales en el hogar y en los primeros años en el sistema escolar. En el caso de Argentina, su pertinencia se manifiesta cuando se considera que pese a haberse alcanzado una cobertura prácticamente completa de la escolaridad básica en el nivel primario para los niños de entre seis y 11 años de edad (mayor a 99\%) (Instituto Nacional de Estadísticas y Censos [INDEC], 2010), los datos también muestran que para el total del país, la tasa de sobreedad en la escuela primaria correspondiente al año 2013 fue de 15,6 \% y que los mayores índices de repitencia se observan en primer grado (3,6 \% en primer grado vs. 2,8 \% para toda la primaria en el año 2012) (Dirección Nacional de Información y Estadística de la Calidad Educativa [DINIECE], 2012/2013). El conocimiento del desarrollo del vocabulario y del discurso en los contextos de uso y vida cotidiana resulta necesario en el entorno escolar tanto para una apropiada comprensión del desempeño infantil en este entorno como para el diseño de situaciones de enseñanza que recuperen dimensiones de los contextos socioculturales y de los patrones de interacción en la construcción de entornos potentes para el desarrollo lingüístico y cognitivo infantil.

Teniendo en cuenta las consideraciones planteadas, en el presente trabajo se realiza una revisión de investigaciones sobre el desarrollo del vocabulario y del discurso narrativo y argumentativo; en particular, de investigaciones que muestran la relevancia de la interacción social en estos desarrollos. En el marco que proporcionan trabajos de investigación de relevancia de la región, de Estados Unidos y de Europa, se presenta una revisión de investigaciones llevadas a cabo por nuestro grupo de trabajo en Argentina. Asimismo, seleccionamos de nuestras investigaciones fragmentos de intercambios registrados en el contexto escolar y en los hogares de niños de sectores medios y de niños que viven en poblaciones urbanas en situación de pobreza y los analizamos con el objeto de ilustrar las oportunidades que estos intercambios generan para el desarrollo del vocabulario y del discurso.

\section{Desarrollo}

\section{El desarrollo de vocabulario}

Los resultados de investigaciones recientes (Hart \& Risley, 1995; Hoff, 2006; Hurtado, Marchman \& Fernald, 2008; Küntay \& Slobin, 2002; Lieven, 2010; Moreno-Manso, García-Baamonde, Blázquez-Alonso, 2010; Tomasello, 2003; Veneziano \& Parisse, 2010) sugieren que muchas de las características del desarrollo del lenguaje infantil se hallan ligadas a las experiencias lingüísticas tempranas. Sin embargo, Hoff y Tian (2005) y Lieven (2010) han señalado que de los diferentes dominios del lenguaje, el vocabulario es el más sensible al input -el lenguaje que el niño escucha- y a la experiencia infantil. Las investigaciones mostraron que la frecuencia y el patrón de ocurrencia de las palabras en el input influye en la composición del vocabulario (Choi \& Gopnik, 1995; Harris, Barret, Jones \& Brookes, 1988; Rosemberg, Alam \& Stein, 2014a).

Los niños aprenden palabras y significados para los conceptos y referentes a los que se hallan expuestos y que les interesan. En la medida en que las experiencias infantiles y la exposición al lenguaje en el mundo difiere, 
es esperable que el vocabulario infantil también difiera. Los trabajos mencionados proporcionan evidencia de que las diferencias entre los niños en la amplitud del vocabulario resultan de las oportunidades que el ambiente tempranamente les ofrece y no, principalmente, de sus capacidades individuales (Hart \& Risley, 1995; Hoff, 2006; Weizman \& Snow, 2001). A los cuatro años de edad, la amplitud del vocabulario infantil está en gran medida determinada por la cantidad, la diversidad y la calidad de las palabras que usan los padres en los intercambios cotidianos (Weizman \& Snow, 2001). Algunos estudios han mostrado que a pesar de que la calidad del entorno léxico difiere entre familias de un mismo grupo social, las diferencias entre grupos socioeconómicos son muy marcadas (Hart \& Risley, 1995; Hoff, 2006). Hoff (2006) encontró que el mejor predictor del desarrollo del vocabulario es la Longitud Media de la Emisión (Mean Length of Utterance, MLU) que el niño escucha. Por su parte, Pan, Rowe, Singer y Snow (2005) mostraron que la diversidad del vocabulario materno tiene un mayor efecto sobre el vocabulario infantil que simplemente la cantidad. Estos trabajos señalan la utilidad de analizar el entorno léxico al que están expuestos niños de distintos grupos sociales en el hogar.

En una investigación longitudinal con niños hispanos en Estados Unidos, Hurtado et al. (2008) analizaron la relación entre el habla de las cuidadoras -principalmente las madres- y el desarrollo de las habilidades de reconocimiento de palabras y el incremento en el vocabulario por parte de los niños en el período comprendido entre los 18 y 24 meses. Las características del habla materna -medida en términos del número de enunciados, la cantidad total de palabras, la cantidad de tipos distintos de palabras y el largo promedio de emisión cuando el niño tenía 18 meses, correlacionaron con la amplitud del vocabulario y la rapidez en el procesamiento de palabras a los 24 meses. Los resultados también mostraron que el procesamiento del lenguaje y el conocimiento del vocabulario se hallan sinérgicamente relacionados.

Tomasello (2003) señaló que no solo el lenguaje al que está expuesto el niño importa; sino también los aspectos pragmáticos de la situación en la que este se encuentra con una palabra estructura el proceso de aprendizaje. Carpenter, Nagell y Tomasello (1998) observaron una correlación entre la cantidad de tiempo en que los niños compartían con sus madres la atención en objetos y actividades y la amplitud de su vocabulario. Tomasello et al. (2007) mostraron que, antes de que emerja el lenguaje, los niños de entre 12 y 14 meses se comunican de manera cooperativa a través de gestos de señalamiento con el dedo, dentro de un marco de atención compartida. Estudios más recientes muestran que los gestos no solo preceden sino también predicen y, presumiblemente, facilitan el desarrollo léxico y sintáctico en el niño (Özçalişkan \& Goldin-Meadow, 2005).

Otra serie de trabajos han analizado en el desarrollo del lenguaje infantil el impacto de la confluencia del habla dirigida al niño y la contingencia como característica de los intercambios conversacionales (Veneziano \& Parisse, 2010). Las contingencias conversacionales permiten que los niños se focalicen en los significados a ser expresados y en las posibles relaciones entre los significados y las formas. Las relaciones establecidas en las contingencias conversacionales son más poderosas para orientar y establecer las producciones infantiles específicas que el input considerado en general. En efecto, las contingencias conversacionales proporcionan relaciones reforzantes cercanas temporalmente en un momento en el que la atención del niño se encuentra focalizada en el verbo, bien porque lo ha pronunciado recién o bien porque se halla a punto de pronunciarlo y cuando la forma y su significado están contextual o discursivamente ancladas.

El concepto de serie de variación de Küntay y Slobin (2002), al igual que las investigaciones acerca de la contingencia semántica recuperan la línea de trabajos psicolingüísticos de la década de los años 1970 
VOCABULARIO, NARRACIÓN Y ARGUMENTACIÓN EN LOS PRIMEROS AÑOS DE LA INFANCIA Y LA NIÑEZ.

sobre las reestructuraciones en el discurso de la díada madre - niño (Nelson, 1977; Snow \& Ferguson, 1977). Específicamente, se conceptualizan ciertas relaciones sucesivas entre el habla de los adultos y los niños pequeños. A través de sustituciones lexicales y reconceptualizaciones, la adición y la omisión de términos específicos y el reordenamiento de las estructuras sintácticas que componen las emisiones, los adultos contribuyen al desarrollo morfológico, léxico y sintáctico de los niños. Las series de variación pueden tener distintas funciones pragmáticas: funciones de control de la actividad del niño; ideacionales, cuando proporcionan información o pueden implicar la elicitación de información. En ellas se repite la mayor parte del contenido de las emisiones de modo tal que se maximizan las oportunidades de comprensión de los niños de las variaciones producidas.

En su conjunto, estas investigaciones proporcionan evidencia adicional a las posturas que consideran que la atención mutua y la participación en los intercambios conversacionales son una esencial fuente de influencia en el desarrollo lingüístico infantil (Saxton, 2000; Snow, 1983; Veneziano, 2005). El habla dirigida al niño posee su mayor poder predictivo en el contexto de la interacción a través de las expansiones, las reformulaciones y las correcciones (Chouinard \& Clark, 2003; Saxton, 2000) de las emisiones de los niños, que retoman o imitan las emisiones de los adultos (Snow, 1983) y, en general, en conversaciones caracterizadas por la atención mutua y la capacidad de respuesta (Rosemberg, Alam \& Stein, 2014b; Rosemberg \& Silva, 2009; Tomasello \& Farrar, 1986; Veneziano, 2005).

En estudios que hemos realizado muy recientemente (Rosemberg et al., 2013; Rosemberg, Stein, Alam \& Piacente, 2013) atendieron a la comparación preliminar entre la cantidad, la diversidad y la abstracción del vocabulario (sustantivos, adjetivos y verbos) que configura el entorno lingüístico en los hogares de niños de cuatro años de sectores medios con padres con educación universitaria, de niños que viven en poblaciones en situación de pobreza y de niños de comunidades indígenas suburbanas qom cuyos padres poseen educación primaria o menor. Se observó que en estos aspectos los entornos de los que participan los niños de sectores medios y de las comunidades qom se diferencian significativamente del entorno del que participan los niños que viven en poblaciones en situación de pobreza. En el grupo de los niños de las comunidades qom se observó, además, una interacción significativa entre el número de interlocutores y la cantidad y diversidad de vocabulario en el entorno. En otro estudio (Rosemberg et al., 2014b), se identificó que los niños de sectores medios empleaban un número significativamente mayor de términos temporales y secuenciales (cantidad y diversidad) en la producción de narrativas futuras espontáneas que los niños de poblaciones urbano marginadas y que ello correlacionaba con diferencias en la cantidad y la diversidad de los términos temporales y secuenciales que los niños escuchaban en los intercambios en el medio familiar. Más allá de la frecuencia en el input, se identificaron ciertas características interaccionales y discursivas de los intercambios en el hogar, que estaban asociadas con las diferencias entre los grupos. En este sentido, en la población de sectores medios, el uso de términos temporales y secuenciales tenía lugar con mayor frecuencia que en las poblaciones urbano marginadas, en el marco de intercambios focalizados en la anticipación de eventos futuros y en los que el habla estaba dirigida el niño, como se observa en el siguiente ejemplo:

(1) [Canela (niña) está jugando en su cuarto, su mamá entra, le pregunta qué está haciendo y le propone otras actividades para más tarde.]

1 MADRE: — ¿Qué estás haciendo?

2 NIÑA: - Jugando

3 MADRE: - Bueno (.) te dejo un ratito sola jugando y yo más tarde vengo y

4 preparamos las cosas (.) primero vamos a armar la mochila 


\section{El desarrollo del discurso narrativo}

Los primeros relatos infantiles constituyen reconstrucciones lingüísticas de los eventos habituales en la vida cotidiana (Fivush, Haden \& Reese, 2006; Nelson, 1996). Progresivamente, los niños pueden narrar un hecho particular situado en el tiempo, que constituye una desviación de un evento general. Para narrar una experiencia personal pasada es necesario retrotraerse temporalmente, recuperar el evento pasado y representarlo verbalmente en función de las relaciones que se establecen en la lengua. La narración es el modo natural en el que las categorías lingüísticas se imponen -organizan y representan- a las estructuras conceptuales derivadas de la experiencia. El sistema conceptual está preparado para segmentar la experiencia en secuencias de acciones que tienen un objetivo, están organizadas temporalmente y localizadas en el espacio (Bruner, 1986; Nelson, 1996). El discurso narrativo refleja, precisamente, esas secuencias de acciones (Labov, 1972).

Las conversaciones sobre eventos futuros, al igual que los intercambios en torno a eventos pasados, constituyen contextos importantes para que los niños entiendan el modo en el que la secuencia de eventos y los conceptos temporales se relacionan en el lenguaje y el pensamiento (Hudson, 2006; Nelson 1996). El lenguaje empleado por las madres para describir los eventos futuros es más complejo, en tanto implica el uso de un lenguaje hipotético (Hudson, 2006). En las conversaciones sobre el futuro, los niños no pueden recurrir directamente a la referencia a un hecho previo, sino que con base en conocimientos de eventos generales o de situaciones pasadas similares, deben, en el marco de la interacción, construir una representación posible del evento futuro (Hudson, 2006). Alrededor de los tres años de edad, el lenguaje empleado por los niños para hablar sobre el futuro refleja su capacidad para anticipar situaciones (Atance \& O’Neill, 2005).

Las narrativas personales de eventos pasados y futuros proporcionan la base para la elaboración de narrativas de ficción (Nelson, 1996). Los relatos de ficción implican la posibilidad de acceder a formas de pensar diferentes, personas, seres tiempos y espacios alejados de la propia realidad. El desarrollo de las narraciones -en tanto forma de discurso y de pensamiento- sobre eventos pasados, futuros y de ficción, requiere habilidades para elaborar un discurso coherente: el empleo de conexiones temporales y causales que permitan articular los eventos (Alarcon-Neve \& Auza-Benavides, 2015); la capacidad para distinguir lo canónico y lo no canónico; el establecimiento de relaciones entre los personajes; el mantenimiento de la referencia de los participantes; la creación de conflictos y desviaciones y su posterior resolución (Hess, 2003 citado en Hess \& Álvarez, 2010; Hess \& Prado, 2013; Nelson, 1996); y la atención a las señales provistas por los interlocutores para negociar el grado de explicitud y de contextualización del relato (Calsamiglia \& Tuson, 2007).

Estas habilidades que permiten construir una narrativa compleja se desarrollan como producto de la organización cognitiva y lingüística propia del ser humano, también como resultado de los modelos culturales que se expresan a través de formas lingüísticas y de la interacción social (Hess \& Alvarez, 2010; Hess \& Prado, 2013; Nelson, 1996, 2007). Por ello, una serie de trabajos atendieron a la producción de relatos de experiencias personales pasadas en la interacción niño-adulto (Carmiol \& Sparks, 2014; Fivush et al., 2006; Nolivos \& Leyva, 2013). Algunos de estos trabajos identificaron, en familias de sectores medios, distintos estilos de interacción maternos (Fivush et al., 2006): un estilo pragmático, en el que el adulto pregunta por información aislada, y otro elaborativo (o narrativo), en el que el andamiaje contribuye a la organización temporal y causal del relato infantil y a la evaluación del evento narrado. Investigaciones realizadas con población hispana mostraron, en consonancia con trabajos que señalan que los estilos de crianza difieren en función de la cultura (Auza-Benavides, 2013; Rosabal-Coto, 2012), que los estilos 
VOCABULARIO, NARRACIÓN Y ARGUMENTACIÓN EN LOS PRIMEROS AÑOS DE LA INFANCIA Y LA NIÑEZ.

de interacción maternos pragmático (o repetitivo) y elaborativo no son universales (Caspe \& Melzi, 2008; Silva \& McCabe, 1996). En estas poblaciones, los adultos intentan mantener la conversación para que los niños recuperen diversos eventos pasados y priorizan los detalles, la descripción y la evaluación (Silva \& McCabe, 1996).

En la mayoría de los trabajos mencionados, se recurrió a diseños cuasiexperimentales que restringieron el análisis a las narrativas producidas en la díada madre-niño. De ahí que en nuestras investigaciones realizadas con corpus de habla registrados en hogares de niños de cuatro años de distintos grupos sociales intentamos dar cuenta de cuestiones como por ejemplo: cuáles son las características que asumen en los primeros años los relatos sobre eventos pasados y futuros que los niños producen en interacción con diferentes interlocutores; qué función cumplen estas narrativas en la interacción social y cómo se construyen en los intercambios colaborativos; cuál es el papel de la narrativa como contexto para otros aprendizajes, por ejemplo, de vocabulario; todas ellas constituyen cuestiones que, desde hace varios años, motivan las investigaciones de nuestro grupo de investigación.

El análisis comparativo que realizamos en nuestras investigaciones entre las narrativas de eventos pasados producidas por los niños que viven en poblaciones en situación de pobreza, y las producidas por los niños de sectores medios y de comunidades indígenas qom no mostró diferencias significativas entre los grupos en el marco de referencia temporal empleado ni en la cantidad de términos temporales y secuenciales. Se observó, en cambio, que los niños de sectores medios empleaban un número significativamente mayor de conectores causales (Rosemberg, Alam \& Stein, 2012). Por su parte, la comparación de los relatos futuros de los niños de los tres grupos sociales mostró que en los tres grupos, las narrativas autorreguladas eran más frecuentes que las heterorreguladas. Se encontraron diferencias entre ellos en la adopción de un marco de referencia hipotético y en el vocabulario empleado, en particular en el uso de términos temporales y en los conectores causales (Rosemberg, Alam \& Stein, 2011).

El análisis de las narrativas producidas por niños pequeños, que viven en poblaciones pobres en situaciones de interacción en su medio familiar, mostró que, en situaciones autorreguladas, los niños pueden producir narraciones de experiencias pasadas complejas que se insertan funcionalmente de distintos modos en los contextos interaccionales (Rosemberg, Silva \& Stein, 2010). Se observó que el grado de textualización logrado en estas narraciones depende de la función que la narrativa cumple en la interacción. Por ejemplo, las narraciones más complejas se producen en contextos en los que la narrativa cumple una función de evidencia factual en el marco de una conversación polémica.

(2) [Tomás quería jugar con Milagros y ella se negaba.]

1 MILAGROS: - Yo no me junto más con vos (.) con la Estelita sola me junto

2 TOMÁS: - Si ella se quiere juntar con vos (.) ella estaba mirando las estrellas

3 así acostada (.) vos fuiste y le pisaste toda la cabeza.

4 MILAGROS: - ¿A dónde? ¿A ella? yo nunca le piso a las amigas mías.

5 TOMÁS: - Sí, sí (.) porque ella estaba con la Soledad mirando las estrellas.

6 MILAGROS: - Eso es mentira Tomás (.) yo no me junto más con ellas (.) con la

$7 \quad$ Soledad, con nadie de ellas (.) con las piojentas yo no me junto.

8 TOMÁS: -Caminando, hiciste que no la veías y le pisaste toda la cara.

9 MILAGROS: -Porque ella me peleaba (.) ella me peleó acá (.) que dejó morado (.)

10 y después yo no fui más la amiga de ella (.) después empecé a ser la amiga (.)

11 mi mamá dijo que le perdone. 
Como se observa en el intercambio, en su intento por convencer a Milagros de que acepte jugar con él, Tomás afirma que la niña con la que Milagros quiere jugar -Estelita- no querrá jugar con ella y justifica esa afirmación mediante la referencia a un evento pasado -líneas dos y tres. Ante la evidencia presentada por Tomás en la narración, Milagros relata como contrapartida el evento que justifica su accionar -líneas nueve a 11. Ambos niños emplean una serie de recursos con el fin de construir la verosimilitud de sus relatos: ofrecen detalles -que dejó morado-, aparecen referencias deícticas en espacios clave -ella me peleó acá-, respetan la secuencia temporal y presentan conectores para afirmarla -y después yo no fui más la amiga de ella (.) después empecé a ser la amiga- y propician la aparición de marcadores que no permiten albergar dudas con respecto a las relaciones causales -porque ella me peleaba.

El análisis de los intercambios espontáneos mostró, asimismo, que los niños de esta edad poseen el control productivo de recursos lingüísticos -condicionales, conectores- para elaborar una narrativa en la que estratégicamente se contemplan escenarios y alternativas de acción futura (Rosemberg et al., 2011) como se observa en el siguiente ejemplo:

(3)

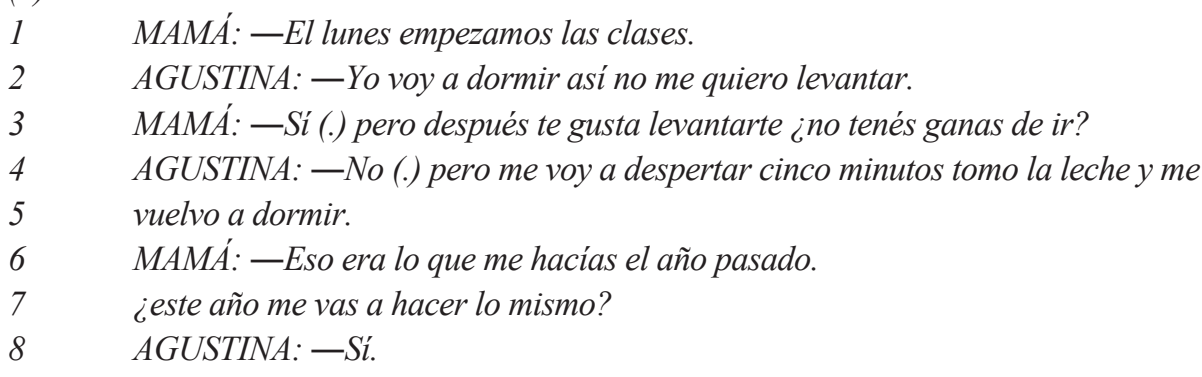

Tampoco abundan estudios en los que se haya analizado, en la producción de narrativas infantiles en situaciones de interacción, cómo se integra o yuxtapone (Goodwin, 2000) información de diferentes campos semióticos. Sin embargo, tanto los ítems léxicos y las construcciones sintácticas como la entonación, la posición corporal, los gestos y la mirada son partes constitutivas de la narración. Por ello, algunas investigaciones enmarcadas en el análisis de la conversación y la sociolingüística interaccional (Goodwin, 2000; Michaels, 1988) han analizado estos aspectos. Los trabajos realizados desde un enfoque multimodal (Andersen, Scheuer, PérezEcheverría \& Teubal, 2009; Kress, 2010) mostraron que la inclusión de diversos modos semióticos es un facilitador para la producción narrativa (Hull \& Nelson, 2005; Thomas, 2012; Vasudevan, Schultz \& Bateman, 2010).

Son escasas y recientes las investigaciones que se focalizan específicamente en la producción de narrativas en la interacción entre pares. Nicolopoulou (2002) mostró, en un programa de intervención con niños preescolares de sectores urbano marginados, cómo la colaboración entre pares puede incidir en el vocabulario y el desarrollo narrativo de los niños pequeños. Asimismo, Küntay (2009) mostró que durante la narración de experiencias pasadas los niños se apoyan en las narrativas de sus compañeros para producir sus propios relatos. En estos casos, la narrativa solía ser estructuralmente más elaborada que la narrativa precedente.

Algunos de nuestros estudios (Alam, 2015; Alam \& Rosemberg, 2015) analizaron el proceso de construcción interaccional de narrativas entre niños. Se observó que los niños adoptaban diferentes roles narrativos -tutor, narrador y 
VOCABULARIO, NARRACIÓN Y ARGUMENTACIÓN EN LOS PRIMEROS AÑOS DE LA INFANCIA Y LA NIÑEZ.

audiencia- que se configuraban a partir de una yuxtaposición de campos semióticos -verbales, gestuales y proxémicos. Cuando los niños narraban en interacción con un niño de igual edad, en la mayoría de los casos, ambos niños adoptaban roles de narrador dando lugar a un proceso de negociación que les permitía coconstruir la narrativa (Alam \& Rosemberg, 2015), tal como se muestra en el siguiente relato de experiencia pasada producido entre Sebastián y Gabriel:

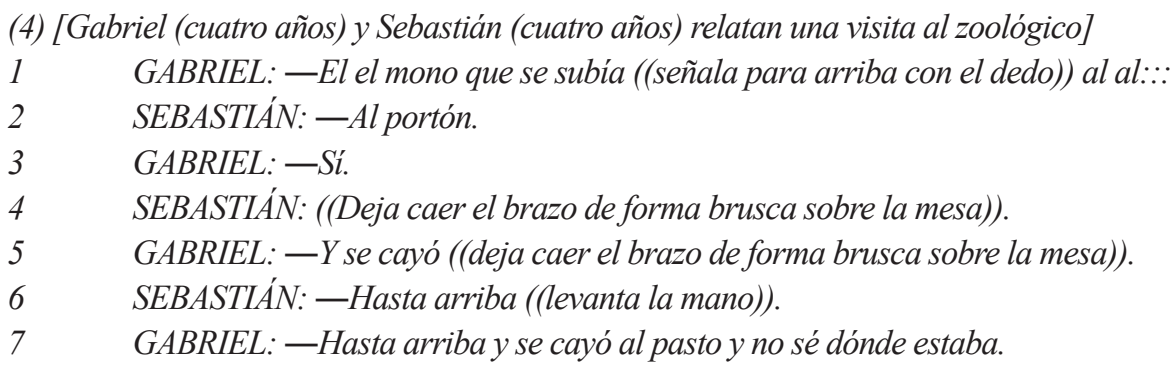

En la construcción de la secuencia narrativa cada uno de los niños aporta un elemento nuevo -verbal o gestualy su compañero lo retoma en el turno siguiente y le agrega nueva información. Por ejemplo, Gabriel relata la acción del mono -el el mono que se subía- pero la repetición y el alargamiento que produce a continuación -al al:::- parecería mostrar que le falta información. Sebastián entonces completa la información -al portón-. A continuación, Sebastián deja caer el brazo de forma brusca sobre la mesa representando probablemente lo que le ocurrió al mono, pero no incluye información verbal. En el turno siguiente, Gabriel repite el movimiento y le agrega la información verbal -y se cayó.

En otras situaciones, la adopción de roles de narrador por parte de ambos niños daba lugar a disputas. En estos casos, los niños apelaban a recursos discursivos, tales como conectores adversativos para sostener su rol y así poder desplegar su narrativa (Alam \& Rosemberg, 2013).

\section{El desarrollo del discurso argumentativo}

El estudio de la argumentación ha estado principalmente focalizado en el discurso adulto, mayoritariamente a partir de fuentes escritas (Stein \& Albro, 2001). Comparativamente con el desarrollo que poseen las diversas teorías que atienden al argumentar adulto, el interés por la argumentación infantil es reciente. Los primeros trabajos la denominan protoargumentación, en tanto carece de ciertas operaciones racionales consideradas específicas del argumentar adulto (Crespo, 1995; Peronard, 1991; Silvestri, 2001). En la protoargumentación se observan enunciados que buscan convencer al interlocutor de la verdad de aquello que se afirma o niega, justificar una acción o un pedido, sin la estructura completa razón-conclusión (Peronard, 1991; van Dijk, 1983). Esta conceptualización implica la consideración de la argumentación como una actividad guiada por objetivos intelectuales, con énfasis en la validación de la lógica o de la verdad de las pruebas (Stein \& Albro, 2001; Zadunaisky-Ehrlich \& Blum-Kulka, 2010). Sin embargo, la argumentación puede ser definida de múltiples maneras, dependiendo de las metas que se estipulen como la búsqueda de una racionalidad que fundamente justificaciones y refutaciones, también como un medio para la resolución de conflictos y la búsqueda de consensos en el marco de la interacción social (Voss \& Van Dyke, 2001). Resulta de importancia, pues, conocer las características que adopta la argumentación infantil, más aun cuando diversos estudios han señalado la importancia del entorno -directo e indirecto- para su desarrollo (Faigenbaum, 2012; Silvestri, 2001).

Una serie de trabajos conceptualizan la producción infantil recurriendo a las categorías de las teorías de la argumentación desarrolladas a partir del discurso adulto (Crespo, 1995; Molina, 2012). Otros trabajos, en cambio, atienden a 
la argumentación infantil desde una concepción que considera como punto de partida la lógica del razonamiento infantil (Cobb-Moore, Danby \& Farell, 2009; Dunn \& Munn, 1987). Por ejemplo, Dunn y Munn (1987) encontraron que en situaciones de disputa en la familia los niños de 18 meses sostienen su punto de vista a través de una "estructura circular", con justificaciones basadas en sus sentimientos, para luego, hacia los tres años, comenzar a utilizar reglas sociales y consecuencias materiales en apoyo a sus posiciones. Por su parte, Pontecorvo y Arcidiacono (2010) analizaron el razonamiento desplegado por niños de cuatro años durante conversaciones argumentativas que tienen lugar en diferentes contextos. En el marco de la producción de una narrativa en el jardín de infantes, los niños negocian el curso de la acción dramática ponderando consecuencias negativas de las diversas propuestas y señalando condiciones hipotéticas y mediante el uso de formas contrafactuales. En las conversaciones durante las comidas en el hogar, los padres proveen justificaciones de reglas de la vida cotidiana mediante estructuras condicionales y formas negativas, que luego también son usadas por los niños.

En el marco de nuestras investigaciones, el análisis de las disputas durante situaciones lúdicas en el hogar y el jardín de infantes a los que asisten niños de poblaciones en situación de pobreza dio lugar a la elaboración de categorías que conceptualizan las estrategias de argumentación que niños de tres, cuatro y cinco años utilizan para sostener sus puntos de vista (Migdalek \& Arrúe, 2013; Migdalek et al., 2014). Los resultados mostraron que los niños sostienen su posición y logran que sus compañeros lleven a cabo determinadas acciones recurriendo a estrategias argumentativas verbales de reiteración, anticipación, generalización, narración, descripción, cortesía, apelación a la autoridad y propuesta alternativa. Migdalek et al. (2014) encontraron que a los cuatro y cinco años son significativamente más las oportunidades en las que los niños emplean estrategias argumentativas que aquellas en las que simplemente se oponen a un punto de vista. También, se ha observado un comportamiento sistemático de ciertos componentes no verbales producidos por los niños para sostener los puntos de vista en las disputas y persuadir a los interlocutores. Esto ha permitido la generación inductiva de un sistema de categorías de estrategias argumentativas no verbales que se yuxtaponen a las estrategias verbales mencionadas (Migdalek \& Rosemberg, 2013; 2014): reforzadores del punto de vista, recursos destinados a proveer evidencia y recursos destinados a conmover. Dichas estrategias configuran conjuntamente con las estrategias verbales la fuerza argumentativa de los enunciados de los niños en las disputas. En el siguiente intercambio, registrado durante la actividad de juego en rincones en una sala de tres años de un jardín de infantes, tiene lugar una disputa por los roles que desempeñarán los niños en la dramatización del cuento Los tres chanchitos.

(5)

1 LAUTARO:-VAMos a jugar a los tres chan:chitosl vos sos el lobol

2 vos sos el lobo ¿dale? ((Señala a Carlos con un bloque)) [La mirada de

3 Lautaro y la de Alejo se dirigen a Carlos].

4 CARLOS: ((Afirma con la cabeza))

5 MAESTRA:-Bueno, armale la casa a ver si el lobo lo puede tirarl.. o nol

6 LAUTARO: yo soy el- . yo soy el-.

7 ALEJO: - NO::YO soy el lobo 1

8 LAUTARO: - NO:/ [Niega con la cabeza] él es el lobo ((Señala a Carlos))

9 ALEJO: NO::-Yo QUIERO ser el LO/-BO/ [Con vehemencia].

10 LAUTARO: - Cada unol ((Muestra el dedo índice levantado)).

11 MAESTRA: - un turno cada unol.

12 LAUTARO:-Dalel. 
VOCABULARIO, NARRACIÓN Y ARGUMENTACIÓN EN LOS PRIMEROS AÑOS DE LA INFANCIA Y LA NIÑEZ.

Para comenzar la dramatización, Lautaro organiza el juego asignando los roles que él y sus compañeros -Carlos y Alejo- desempeñarán. Lautaro designa en los primero turnos a Carlos como "el lobo" -líneas uno a cuatro- pero Alejo se opone, dando inicio a la disputa -NO::YO soy el lobo 1 . La oposición se estructura con la expresión del punto de vista en la que se observan estrategias argumentativas no verbales de refuerzo, como el aumento de la intensidad sobre la partícula negativa junto con un alargamiento vocálico -NO::-. Lautaro insiste en su posición a través de su reiteración en la que también recurre a estrategias de refuerzo del punto de vista, que consisten en este caso no solo en el énfasis y el alargamiento vocálico de la partícula negativa, sino también en su yuxtaposición con el movimiento de su cabeza hacia los lados, negando. Ante la firmeza de Lautaro en su postura, Alejo contraargumenta a partir de una estrategia de descripción, que consiste en este caso en referir su deseo de ser el lobo — NO:: yo QUIERO ser el $L O /-B O /$. Tal enunciado se conforma, además, con la estrategia no verbal destinada a conmover en tanto es emitido con una entonación vehemente que se evidencia en el aumento de la intensidad y la segmentación en la emisión de la palabra lobo. Tal resistencia produce que, finalmente, Lautaro apele a la estrategia de propuesta alternativa como forma de resolver la disputa -cada unol. Entonces, la maestra valida la propuesta al reiterarla, -un turno cada unol- y el juego da comienzo. Este tipo de comportamiento, la búsqueda de la solución a un desacuerdo por la vía argumentativa, parece estar favorecido por el contexto institucional del jardín de infantes y sus normas, tal como lo evidencian los resultados de nuestros últimos trabajos (Migdalek, Rosemberg \& Arrúe, 2015). Cabe señalar, también, que el uso de estrategias argumentativas conduce, en muchos casos, a que los niños recurran a conectores causales, consecutivos y adversativos.

\section{Consideraciones finales}

Los resultados de nuestros estudios, al igual que los de investigaciones antecedentes (Nelson, 2007; Tomasello, 2003), señalan la importancia de analizar de modo articulado el desarrollo del vocabulario y del discurso narrativo y argumentativo. Como puede observarse en los intercambios analizados, los desarrollos tienen lugar, simultánea y entrelazadamente, en situaciones de interacción social en las que el niño comparte con otros niños y adultos las actividades que configuran la vida cotidiana en su hogar y su comunidad y en el entorno escolar (Nelson, 1996, 2007; Tomasello, 2003). Por ejemplo, los estudios que analizaron las disputas que tienen lugar en situaciones de juego en el hogar y el jardín de infantes muestran cómo las descripciones y las narraciones son empleadas en estos contextos por niños de tres, cuatro y cinco años, como estrategias argumentativas verbales para defender su punto de vista (Migdalek \& Arrúe, 2013; Migdalek \& Rosemberg, 2013; Migdalek et al., 2014) y para concretizar esas estrategias ponen en juego y, por tanto, tienen la oportunidad de desarrollar conectores de diversa índole.

Al igual que estudios previos (Fivush et al., 2006; Hudson, 2006), los resultados de nuestros trabajos han puesto de manifiesto que las conversaciones que giran en torno a distintos tipos de narrativas -eventos pasados, futuros y de ficción- generan diferentes contextos para los aprendizajes infantiles; por ejemplo, el empleo de vocabulario preciso y diverso (Rosemberg, 2013) y, en particular, el uso de términos temporales y secuenciales (Nelson 1996; Hudson, 2006; Rosemberg et al., 2014b) y de conectores causales (Nelson, 1996; Rosemberg et al., 2011, 2012).

Cabe señalar, además, la relevancia de una aproximación ecológica a las situaciones de interacción en el hogar y en el medio escolar en tanto que ella contribuye a revelar características multimodales, que configuran las oportunidades que estas situaciones ofrecen para el uso del lenguaje infantil, así como a las características que este uso adopta (Hull \& Nelson, 2005; Thomas, 2012; Vasudevan et al., 2010). 
En este sentido, los trabajos que hemos reseñado en el presente artículo han mostrado cómo durante los intercambios conversacionales, los interlocutores (madres, maestras y niños) yuxtaponen a sus emisiones verbales, información multimodal (Alam, 2015; Alam \& Rosemberg, 2013, 2014, 2015; Menti \& Alam, 2014; Menti \& Rosemberg, 2013; Migdalek \& Rosemberg, 2013, 2014). Los gestos, la dirección de la mirada, la entonación, la posición corporal y los señalamientos de objetos presentes en el entorno configuran junto con las emisiones verbales situaciones de aprendizaje que promueven el desarrollo del vocabulario (Menti \& Alam, 2014; Menti \& Rosemberg, 2013; Rosemberg, 2013) en el marco de la elaboración compartida de discurso (Alam \& Rosemberg, 2014; Goodwin, 1997).

La relevancia de esos resultados es evidente cuando se considera que el aprendizaje temprano de estos conocimientos y habilidades lingüísticas, discursivas y cognitivas adquiere un papel muy importante en el proceso de alfabetización durante la transición a la escolaridad formal (Simons \& Murphy, 1988). Asimismo, promueve el desarrollo de comprensión y producción de otros géneros discursivos (Reese, Yan, Jack \& Hayne, 2010). En conjunto, los resultados de los estudios reseñados poseen importantes implicancias pedagógicas, en tanto plantean la necesidad de atender, desde una perspectiva multimodal, al contexto de interacción social en el que se insertan tempranamente las palabras infantiles cuando los niños comienzan a producir discursos que les permiten realizar su intención comunicativa. En el entorno de las escuelas infantiles y de los primeros años de la escuela primaria, el conocimiento del vocabulario y del discurso infantil en los contextos de uso y de vida cotidiana puede resultar necesario tanto para ponderar apropiadamente el desempeño infantil como para generar contextos que den lugar a oportunidades para nuevos desarrollos y aprendizajes.

\section{Referencias}

Alam, F. (2015). La construcción interaccional de narrativas de ficción entre niños de distintas edades. Un estudio con niños de poblaciones urbano marginadas. Interdisciplinaria, 32(1), 31-49.

Alam, F. \& Rosemberg, C. R. (2013). El uso de conectores en relatos infantiles de ficción. Diferencias según el contexto interaccional de producción. Lenguas Modernas, 41, 11-32.

Alam, F. \& Rosemberg, C. R. (2014). Narrativas de ficción y de experiencia pasada en la interacción entre niños de distintas edades. Praxis Revista de Psicología, 16(26), 7-29.

Alam, F. \& Rosemberg, C. R. (2015). Un análisis del proceso de construcción interaccional en narrativas de ficción. Papeles de trabajo sobre Cultura, Educación y Desarrollo Humano, 11(1), 1-16.

Alarcon-Neve, L. J. \& Auza-Benavides, A. (2015). Uso y función de nexos en la subordinación y coordinación. Evidencia de dos tareas narrativas de niños mexicanos de primero de primaria. En E.P. Velázquez Patiño \& I. Rodriguez Sánchez (Eds.). Estudios de lingüistica funcional (pp. 223-251). México: Editorial Universitaria UAQ (Col. Academia, Serie Nodos).

Andersen, C., Scheuer, N., Pérez-Echeverría, M. \& Teubal, E. (Eds.), (2009). Representational Systems and Practices as Learning Tools. Rotterdam: Sense Publishers.

Atance, C. M. \& O’Neill, D. K. (2005). Preschoolers' talk about future situations. First Language, 25, 5-18.

Auza-Benavides, A. (2013). La mirada sociocultural en las prácticas narrativas de niños hispanohablantes. Actualidades en Psicología, 27(115), 141-146.

Bruner, J. (1986). El habla del niño. Barcelona: Paidós. 
VOCABULARIO, NARRACIÓN Y ARGUMENTACIÓN EN LOS PRIMEROS AÑOS DE LA INFANCIA Y LA NIÑEZ.

Calsamiglia, H. \& Tusón, A. (2007). Las cosas del decir. Manual de análisis del discurso (2da ed.). Barcelona: Ariel Lingüística.

Carmiol, A. M., Sparks, A. \& Ríos, M. (2013). Evidencia de la relación entre habilidades prelectoras y habilidades narrativas en niños y niñas preescolares costarricenses: Aportes para un enfoque comprensivo de la alfabetización emergente. En A. Auza y K. Hess (Eds.), ¿Qué me cuentas? Narraciones y desarrollo narrativo lingüístico en niños hispanohablantes (pp. 85-110). Querétaro, MX: Universidad Autónoma de Querétaro.

Carmiol, A. M. \& Sparks, A. (2014). Narrative development across cultural contexts: Finding the pragmatic in parent child reminiscing. En D. Mathews (Ed). Pragmatic Development in First Language Acquisition (pp. 279-293). Amsterdam: John Benjamins.

Carpenter, M., Nagell, K. \& Tomasello, M. (1998). Social cognition, joint attention, and communicative competence from nine to 15 months of age. Monographs of the society for research in child development, 63(4), i-vi, 1-143. doi: $10.2307 / 1166214$

Caspe, M. \& Melzi, G. (2008). Cultural variations in mother-child narrative discourse style. En A. McCabe, A. Bailey \& G. Melzi (Eds), Spanish-language narration and literacy (pp. 6-33). Cambridge: Cambridge University Press.

Castellaro, M.A. (2012). Definiciones teóricas y áreas de investigación propuestas desde el constructivismo, en publicaciones latinoamericanas de psicología y educación presentes en la base de datos Redalyc. Liberabit, 18(2), 131-146.

Cekaite, A., Blum-Kulka, S., Grøver, V. \& Teubal, E. (Eds.) (2014). Children's Peer Talk. Learning from Each Other. Cambridge: Cambridge University Press.

Choi, S. \& Gopnik, A. (1995). Early acquisition of verbs in Korean: a cross-linguistic study. Journal of Child Language, 22(3), 497-529. doi: 10.1017/S0305000900009934

Chouinard, M. \& Clark, E. (2003). Adult reformulations of child errors as negative evidence. Journal of Child Language, 30, 637-669. doi: 10.1017/S0305000903005701

Cobb-Moore, C., Danby, S. \& Farrell, A. (2009). Young children as rule markers. Journal of Pragmatics, 41, 1477-1492.

Crespo, N. (1995). El desarrollo ontogenético del argumento. Revista Signos. Estudios de Lengua y Literatura, XVIII(37), 69-82.

Dirección Nacional de Información y Estadística de la Calidad Educativa (DINIECE). (2012/2013). Dirección Nacional de Información y Evaluación de la Calidad Educativa. Recuperado de http://portales.educacion.gov.ar/diniece/

Dunn, J. \& Munn, P. (1986). Sibling quarrels and maternal intervention: Individual differences in understanding and aggression. Journal of Child Psychology and Psychiatry and Allied Disciplines, 27(5), 583-595. doi: 10.1111/j.14697610.1986.tb00184.x

Faigenbaum, G. (2012). El desarrollo de las habilidades argumentativas. En J. A. Castorina \& M. Carretero (Comps.), Desarrollo Cognitivo y Educación [I]. Los inicios del conocimiento (pp. 292-313). Buenos Aires: Paidós.

Farrant, K. \& Reese, E. (2000). Maternal style and children's participation in reminiscing: Stepping stones in autobiographical memory development. Journal of Cognition and Development, 1, 193-225.

Fivush, R., Haden, C. A. \& Reese, E. (2006). Elaborating on elaborations: The role of maternal reminiscing style in cognitive and socioemotional development. Child Development, 77, 1568-1588. doi: 10.1007/s10615-0 06-0065-1 


\section{R. Rosemberg, A. Menti, A. Stein, F. Alam y M. Migdalek}

Goodwin, C. (2000) Action and embodiment within situated human interaction. Journal of Pragmatics, 32, 1489-1522. doi: 10.1016/S0378-2166(99)00096-X

Goodwin, M. H. (1997). Byplay: Negotiating evaluation in storytelling. In G.R. Guy, J. Baugh, D. Schiffrin \& Crawford Feagin (Eds.), Towards a social science of language: Papers in honour of William Labov (pp. 77-102). Philadelphia: John Benjamins.

Goswami, U. (2003). Early phonological development and the acquisition of literacy. In Dickinson, D.K. \& Neuman, S.B. (Eds.), Handbook of early literacy research (pp. 111-115). NY: Guilford Press.

Harris, M., Barrett, M., Jones, D. \& Brookes, S. (1988). Linguistic input and early word meaning. Journal of Child Language, 15, 77-94. doi:10.1017/S030500090001206X

Hart, B. \& Risley, T.R. (1995). Meaningful differences in the everyday experience of young American children. Baltimore: Paul H. Brookes.

Hess, K., \& Álvarez, P. T. (2010). Desarrollo lingüístico y cultura escrita. En G. Calderón \& K. Hess (Eds.), El reto de la lengua escrita en la escuela (pp. 85-114). México: FUNDAp.

Hess, K. \& Prado, M.L. (2013). ¿Te leo un cuento? La lectura de relatos en el desarrollo narrativo de niños en edad preescolar. En A. Auza \& K. Hess (Eds.) ¿Qué me cuentas? Narraciones y desarrollo lingüístico en niños hispanohablantes (pp. 111-140). México: DeLaurel-UAQ-Hospital General Manuel Gea González.

Hoff, E. (2006). How social contexts support and shape language development. Developmental Review, 26, 55-88. doi: 10.1016/j.dr.2005.11.002

Hoff, E. \& Tian, C. (2005). Socioeconomic status and cultural influences on language. Journal of Communication Disorders, 38(4), 271-8. doi:10.1016/j.jcomdis.2005.02.003

Hudson, J. A. (2006). The development of future time concepts through mother-child conversation. Merrill-Palmer Quarterly, 52(1), 70-95. doi: 10.1353/mpq.2006.0005

Hull, G. \& Nelson, M.E. (2005). Locating the semiotic power of multimodality. Written Communication, 22(2), 224-26. doi: $10.1177 / 0741088304274170$

Hurtado, N., Marchman, V. \& Fernald, A. (2008). Does input influence uptake? Links between maternal talk, processing speed and vocabulary size in Spanish-learning children. Developmental Science, 11(6), 31-39. doi: 10.1111/j.1467-7687.2008.00768.x

Instituto Nacional de Estadísticas y Censos (INDEC). (2010). Censo Nacional de Población, Hogares y Vivienda. Recuperado de www.censo2010.indec.gov.ar

Kress, G. (2010). Multimodality: A social semiotic approach to contemporary communication. London: Routledge.

Küntay, A. (2009). Microgenesis of narrative competence during preschool interactions: Effects of the relational context. En A. Aksu-Koç \& S. Bekman (Eds.), Perspectives on human development, family and culture (pp.178-193). Cambridge: Cambridge U. Press.

Küntay, A. \& Slobin, D. I. (2002). Putting interaction back into child language: Examples from Turkish. Psychology of Language and Communication, 6, 5-14. 
VOCABULARIO, NARRACIÓN Y ARGUMENTACIÓN EN LOS PRIMEROS AÑOS DE LA INFANCIA Y LA NIÑEZ.

Labov, W. (1972). Sociolinguistic patterns. Philadelphia, PA: U. of Pennsylvania Press.

Lieven, E. (2010). Input and first language acquisition: Evaluating the role of frequency. Lingua, 120, 2546-2556. doi: 10.1016/j.lingua.2010.06.005

Liszkowski, U., Carpenter, M., Striano, T. \& Tomasello, M. (2006). 12- and 18-month-olds point to provide information for others. Journal of Cognition and Development, 7(2), 173-187.

McCabe, A., Bailey, A. \& Melzi, G. (Eds.). (2008). Spanish-language narration and literacy: Culture, cognition, and emotion. New York: Cambridge University Press. doi: 10.1017/CBO9780511815669

Matthews, D., Behne, T. Lieven, E. \& Tomasello, M. (2012) Origins of the human pointing gesture: a training study. Developmental Science, 15(6), 817-829.

Melzi, G. \& Caspe, M. (2005). Variations in maternal narrative styles during book reading interactions. Narrative Inquiry, $15(1), 101-125$.

Menti, A. \& Alam, F. (2014). Los gestos y la enseñanza de palabras poco familiares: ¿Cuándo emplean las maestras información gestual? Bellaterra Journal of Teaching \& Learning Language \& Literature, 7(1), 17-32.

Menti, A. \& Rosemberg, C. (2013). ¿Cómo se enseñan palabras poco familiares en las clases de ciencias en la escuela primaria? Un estudio en escuelas argentinas. Lenguas Modernas, 41, 95-114.

Michaels, S. (1988). Presentaciones narrativas: una preparación oral para la alfabetización con alumnos de $1^{\circ}$ curso. En J. Cook-Gumperz (Ed.), La construcción social de la alfabetización (pp. 60-73). Barcelona: Paidós.

Migdalek, M. \& Arrúe, J. (2013). Habilidades argumentativas de niños de cuatro años: un análisis de las disputas en situaciones de juego en hogares. En D. Riestra, S. M. Tapia \& M.V. Goicoechea (Eds.), Terceras jornadas internacionales de investigación y prácticas en didáctica de las lenguas y las literaturas (pp. 733-752). Bariloche: Ediciones GEISE.

Migdalek, M. \& Rosemberg, C. (2013). Construcción multimodal de los argumentos de niños pequeños en disputas durante situaciones de juego. Papeles de Trabajo sobre Cultura, Educación y Desarrollo Humano, 9, 1-16.

Migdalek, M. \& Rosemberg, C. (2014). Argumentando cara a cara: Un estudio de las estrategias argumentativas no verbales empleadas por niños en situaciones de juego. Cogency, 6, 91-123.

Migdalek, M. Rosemberg, C. \& Arrúe, J. (2015). Argumentación infantil en situaciones de juego: diferencias en función del contexto. Propuesta Educativa, 44 (2), 79-88.

Migdalek, M., Rosemberg, C. \& Santibáñez, C. (2014). La génesis de la argumentación. Un estudio con niños de tres a cinco años en distintos contextos de juego. Íkala, 19(3), 251-267.

Molina, M. (2012). Estudio de las emociones en la argumentación infantil. RILL Nueva época, Prácticas discursivas a través de las disciplinas, 17(1-2), 140-150.

Moreno-Manso, J. M.; García-Baamonde, M. E., \& Blázquez-Alonso, M. (2010). Desarrollo lingüístico y adaptación escolar en niños en acogimiento residencial. Anales de Psicología, 26(1), 189-196.

Nelson, K. (1977). Facilitating children's syntax acquisition. Developmental Psychology, 13, 101-107.

Nelson, K. (1996). Language in cognitive development. Cambridge: Cambridge University Press. 


\section{R. Rosemberg, A. Menti, A. Stein, F. Alam y M. Migdalek}

Nelson, K. (2007). Young Minds in Social Worlds. Experience, meaning and memory. Cambridge: Harvard University Press.

Nicolopoulou, A. (2002). Peer group culture and narrative development. En S. Blum-Kulka \& C. E. Snow (Eds.), Talking to adults. The contribution of multiparty discourse to language acquisition (pp. 117-152). Mahwah, NJ: Lawrence Erlbaum.

Nicolopoulou, A. (2008). The elementary forms of narrative coherence in young children's storytelling. Narrative Inquiry, $18(2), 299-325$.

Nolivos, V. \& Leyva, D. (2013). Fun and frustration: Low-income Chilean parents reminiscing with their children about past emotional experiences. Actualidades en Psicología, 27(115), 31-48.

Özçalişkan, S. \& Goldin-Meadow, S. (2005). Gesture is at the cutting edge of early language development. Cognition, 96(3), 101-113.

Pan, B., Rowe, M., Singer, J. \& Snow, C. (2005). Maternal correlates of growth in toddler vocabulary production in lowincome families. Child Development, 76(4), 763- 782. doi: 10.1111/1467-8624.00498-i1

Perfetti, C. (2007). Reading ability: Lexical quality to comprehension. Scientific Studies of Reading, 11(4), 357-383. doi: $10.1080 / 10888430701530730$

Peronard, M. (1991). Antecedentes ontogenéticos de la argumentación. En E.L. Traill(Eds.), Scripta philologica: in honorem Juan M. Lope Blanch, 3 (pp. 417-443.). México: UNAM.

Pontecorvo, C. \& Arcidiacono, F. (2010). Development of reasoning through arguing in young children. Cultural-Historical Psychology, 4, 19-29.

Poveda, D., Morgade, M. \& Alonso, B. (2009). Storytellers of children's literature: Ideologies of the audience. Oral Tradition, 24(1), 224-241.

Reese, E., Yan, C., Jack, F. \& Hayne, H. (2010). Emerging identities: Narrative and self from early childhood to early adolescence. In K.C. McLean, \& M. Pasupathi (Eds.), Narrative development in adolescence. Creating the storied self(pp. 23-43). NY: Springer.

Rogoff, B. (2003). The cultural nature of human development. NY: Oxford Univ. Press.

Rosabal-Coto, M. (2012). Creencias y prácticas de crianza: el estudio del parentaje en el contexto costarricense. Revista Costarricense de Psicología, 31(1, 2) 65-100.

Rosemberg, C. R. (2013). El aprendizaje de vocabulario en distintos contextos de relato en el jardín de infantes: Experiencias personales en la ronda y lectura de cuentos. Congreso internacional de educación de la Facultad de Psicología. U. Nac. de La Plata.

Rosemberg, C. R., Alam, F. \& Stein, A. (2011). Children's talk about the future. A study with three social groups from Buenos Aires, Argentina. Society of Research in Child Development Biennial Meeting, Montreal, Canada.

Rosemberg, C. R., Alam, F., \& Stein, A. (2012). Future talk and lexical input. A study with two social groups from Argentina. ELA2012 -Early language acquisition. Lyon.

Rosemberg, C. R., Alam, F. \& Stein, A. (2014a). Factors reflecting children's use of temporal terms as a function of social group. Language, Interaction and Acquisition, 5, 38-61. doi: /10.1075/lia.5.1.02ros 
VOCABULARIO, NARRACIÓN Y ARGUMENTACIÓN EN LOS PRIMEROS AÑOS DE LA INFANCIA Y LA NIÑEZ.

Rosemberg, C. R., Alam, F., \& Stein, A. (2014b). Children interactions in literacy tutoring situations: A study with urban marginalized populations in Argentina. Global Education Review, 1(2), 41-63.

Rosemberg, C. R. \& Silva, M. L. (2009). Teacher-children interaction and concept development in kindergarten. Discourse Processes, 46(6), 572- 591. doi: 10.1080/01638530902959588

Rosemberg, C. R., Silva, M. L. \& Stein, A. (2010). Narrativas infantiles en contexto: un estudio en hogares de barrios urbano marginados de Buenos Aires. Revista del Instituto de Ciencias de la Educación Universidad de Buenos Aires, 28, 135-154.

Rosemberg, C. R., Stein, A. \& Alam, F. (2013). At home and at school: Bridging literacy to children from poor rural and urban communities. En K. Hall, T. Cremin, B. Comber \& L. Moll (Eds.), International Handbook of Research in Children's Literacy, Learning and Culture (pp. 67-82.). Oxford: Wiley-Blackwell.

Rosemberg, C. R., Stein, A., Alam, F. \& Piacente, T. (2013). Lexical Input to Young Children at Home. A Study with Three Social Groups in Argentina. Society of Research in Child Development Biennial Meeting, EEUU.

Saxton, M. (2000). Negative evidence and negative feedback: immediate effects on the grammaticality of child speech. First Language, 20, 221-252. doi: 10.1177/014272370002006001

Schöder, L., Keller, H. \& Kleis, A. (2013). Parent-child conversation in three urban middle-class contexts: Mothers and fathers reminisce with their daughters and sons in Costa Rica, Mexico and Germany. Actualidades en Psicología, 27(115), 49-73

Silva, M. J. \& McCabe, A. (1996). Vignettes of the continuous and family ties: Some Latino American traditions. En A. McCabe (Ed.), Chameleon readers: Teaching children to appreciate all kinds of good stories (pp. 116-136). NY: McGraw-Hill.

Silvestri, A. (2001). La producción de la argumentación razonada en el adolescente: Las falacias de aprendizaje. En Martínez, M.C. (comp.), Aprendizaje de la argumentación razonada (pp. 29-48). Cali: Cátedra UNESCO para la lectura y la escritura en A. Latina.

Simons, H. D. \& Murphy, S. (1988). Estrategias en el lenguaje hablado y en la aptitud de leer. En J. Cook-Gumperz (Ed.), La construcción social de la alfabetización (pp. 85-99). Madrid: Paidós.

Snow, C. (1983). Literacy and language: relationships during the preschool years. Harvard Educational Review, 53(2), 165189. doi: $10.17763 /$ haer.53.2.t6177w39817w2861

Snow, C. E. \& Ferguson, C.A. (1977). Talking to children: Language input and acquisition. Cambridge: Cambridge Univ. Press.

Sparks, A. (2008). Latino mothers and their preschool children talk about the past: Implications for language and literacy. En A. McCabe, A. L. Bailey, \& G. Melzi (Eds.), Spanish language narration and literacy: Culture, cognition, and emotion (pp. 273-295). NY: Cambridge University Press.

Sparks, A., Carmiol, A. \& Ríos, M. (2013). High point narrative structure in mother-child conversations about the past and children's emergent literacy skills in Costa Rica, Actualidades en Psicología, 27(115), 93-111.

Stein, A. \& Rosemberg, C. R. (2012). Redes de colaboración en situaciones de alfabetización familiar con niños pequeños. Interdisciplinaria, 29(1), 95-108. 


\section{R. Rosemberg, A. Menti, A. Stein, F. Alam y M. MigdaleK}

Stein, N. \& Albro, E. (2001). The origins and nature of arguments: Studies in conflict understanding, emotion, and negotiation. Discourse Processes, 32, 113-133. doi: 10.1080/0163853X.2001.9651594

Strasser, K., Del Río, F. \& Larraín, A. (2014). Profundidad y amplitud del vocabulario: ¿Cuál es su rol en la comprensión de historias en la edad pre-escolar? Estudios de Psicología, 34(2),221-225.

Thomas, S. (2012). Narrative inquiry: embracing the possibilities. Qualitative Research Journal, 12(2), 206-221. doi: $10.1108 / 14439881211248356$

Tomasello, M. (2003). Constructing a language: A usage-based theory of language acquisition. Massachusetts: Harvard University Press.

Tomasello, M., Carpenter, M. \& Liszkowski, U. (2007). A new look at infant pointing. Child Development, 78(3), 705-722. doi: 10.1111/j.1467-8624.2007.01025.x

Tomasello, M. \& Farrar, M. (1986). Joint attention and early language. Child Development, 57(6), 1454-1463. doi: $10.2307 / 1130423$

van Dijk, T.A. (1983). La ciencia del texto: un enfoque interdisciplinario. Barcelona: Paidós.

Vasudevan, L., Schultz, K. \& Bateman, J. (2010). Rethinking composing in a digital age: Authoring literate identities through multimodal storytelling. Written Communication, 27(4), 442-468. doi: 10.1177/0741088310378217

Veneziano, E (2005). Effects of conversational functioning on early language acquisition:When both caregivers and children matter. En B. Bokus (Ed.), Studies in the psychology of child language (pp. 47-69). Warsaw: Matrix.

Veneziano, E. \& Parisse, C. (2010). The acquisition of early verbs in French: Assessing the role of conversation and of childdirected input. First Language, 30(3-4), 287-311. doi: 10.1177/0142723710379785

Voss, J.F. \& Van Dyke, J.A. (2001). Argumentation in psychology. Discourse Processes, 32(2-3), 89-111.

Weizman, Z. \& Snow, C. (2001). Lexical input as related to children's vocabulary acquisition: Effects of sophisticated exposure and support for meaning. Developmental Psychology, 37(2), 265-279. doi:10.1037/0012-1649.37.2.265

Wishard Guerra, A. (2008). The intersection of language and culture among Mexican-heritage children three to seven years old. En: A. McCabe, A. L. Bailey, \& G. Melzi (Eds.) Research on the development of Spanish-language narratives. (pp. 143-174) New York: Cambridge University Press.

Wortham, S. (2001). Narratives in action: A strategy for research and analysis. NY: Teachers College Press.

Zadunaisky-Ehrlich, S. \& Blum-Kulka, S. (2010). Peer talk as a 'double opportunity space': The case of argumentative discourse. Discourse Society, 21(2), 211-233.

Recibido: 04 de marzo de 2016 Revisión recibida: 03 de agosto de 2016

Aceptado: 07 de octubre de 2016 
VOCABULARIO, NARRACIÓN Y ARGUMENTACIÓN EN LOS PRIMEROS AÑOS DE LA INFANCIA Y LA NIÑEZ.

\section{Sobre las autoras:}

Celia Renata Rosemberg. Es Doctora de la Facultad de Filosofía y Letras de la Universidad Nacional de Buenos Aires. Funge como Profesora Titular de la Universidad Nacional de Buenos Aires e Investigadora Principal del CONICET (Consejo Nacional de Investigaciones Científicas y Técnicas de Argentina). Sus proyectos de investigación abordan el desarrollo lingüístico y cognitivo infantil de niños de distintos grupos sociales y culturales. Actualmente, labora como directora del CIIPME (Centro Interdisciplinario de Investigaciones en Psicología Matemática y Experimental).

Alejandra Menti. Es Doctora en Ciencias del Lenguaje (Universidad Nacional de Córdoba). Trabaja como investigadora del CIFAL-CONICET (Consejo Nacional de Investigaciones Científicas y Técnicas de Argentina). Investiga las oportunidades de aprendizaje de vocabulario que se proporcionan a los alumnos del nivel inicial y primario en situaciones de interacción. Se desempeña como profesora asistente en la Universidad Nacional de Córdoba.

Alejandra Stein. Es Doctora en Ciencias del Lenguaje (Universidad Nacional de Córdoba). Trabaja como investigadora en CIIPME-CONICET (Consejo Nacional de Investigaciones Científicas y Técnicas de Argentina). Sus estudios se focalizan en el desarrollo del discurso narrativo en niños preescolares. Funge como docente auxiliar en la Universidad de Buenos Aires.

Florencia Alam. Es Licenciada en Letras y Doctora en Educación por la Universidad de Buenos Aires. Trabaja como investigadora en el Consejo de Investigaciones Científicas y Técnicas de Argentina (CONICET) con sede en el CIIPME. Su tema de investigación se centra en la interacción entre niños en la producción de narrativas.

Maia Julieta Migdalek. Es Licenciada en Letras y Doctora en Educación por la Universidad de Buenos Aires. Trabaja como investigadora en el Consejo de Investigaciones Científicas y Técnicas de Argentina (CONICET) con sede en el CIIPME. Su tema de investigación es el desarrollo y la producción argumentativa infantil. Se desempeña como docente auxiliar en la Universidad de Buenos Aires. 


\section{Apéndice}

\section{Codificaciones empleadas en la transcripción}

[ ] comentarios del transcriptor

(( )) acciones no verbales

(0.1) pausa: los números entre paréntesis indican el tiempo de la pausa en segundos

- interrupción

/ entonación ascendente

$\backslash$ entonación descendente

¿? entonación interrogativa

$==$ superposición

MAYÚSCULAS énfasis

: alargamientos sonoros 